\title{
Asociación entre el sobrepeso y la obesidad con el rendimiento académico en estudiantes de secundaria de la ciudad de Mérida, México
}

\author{
Juan Carlos Salazar-Rendón ${ }^{1,2 *}$, Nina Méndez ${ }^{3}$ y Hugo Azcorra ${ }^{4}$ \\ ${ }^{1}$ Departamento de Desarrollo Humano, Secretaría de Educación del Gobierno del Estado de Yucatán; ${ }^{2}$ Dirección de Nutrición, Servicios de Salud \\ de Yucatán; ${ }^{3}$ Universidad Marista de Mérida, Yucatán; ${ }^{4}$ Departamento de Ecología Humana, Centro de Investigación y de Estudios Avanzados del \\ Instituto Politécnico Nacional. Mérida, Yucatán, México
}

\begin{abstract}
Resumen
Introducción: Diversos estudios señalan una relación negativa entre la obesidad y el rendimiento académico en la población infantil. Sin embargo, este tema ha sido poco estudiado en México. El objetivo de este estudio fue analizar la asociación entre el sobrepeso y la obesidad con el rendimiento académico en estudiantes de secundaria de Mérida. Métodos: Se midió el peso y la talla y se calculó el índice de masa corporal (IMC) en una muestra de 566 alumnos de ambos sexos de escuelas secundarias públicas de Mérida. Se clasificó el estado nutricional de los adolescentes en peso normal (-2 - <+1 desviaciones estándar [DE]), sobrepeso $(+1-<2 D E)$ y obesidad $(\geq+2 D E)$ de acuerdo con los valores de referencia de la Organización Mundial de la Salud (OMS). Se utilizó el promedio de calificaciones obtenidas en las asignaturas de lenguaje, matemáticas y ciencias como un indicador del rendimiento académico. Se consideró desempeño mayor cuando el promedio fue $\geq 8$ puntos y menor cuando fue $<8$ puntos. El análisis de asociación entre las variables se realizó mediante modelos de regresión logística. Resultados: La prevalencia conjunta de sobrepeso y obesidad fue del $44 \%$. Al ajustar para el grado escolar, el tipo de escuela, el número de ausencias y la procedencia maya del apellido, las adolescentes con obesidad tuvieron un riesgo significativo de tener desempeño menor en matemáticas (razón de momios [RM]: 3.07; IC 95\%: 1.39-6.80) y lenguaje (RM:2.55; IC 95\%:1.14-5.71) al compararlas con aquellas con peso saludable. Conclusiones: Los resultados sugieren que la obesidad es un factor de riesgo para tener un menor rendimiento académico en las adolescentes de entre 12 y 15 años, pero no entre los adolescentes de la misma edad.
\end{abstract}

Palabras clave: Rendimiento académico. IMC. Obesidad. Adolescentes.

\section{Association between overweight and obesity with school performance in secondary students in Merida, Mexico}

\section{Abstract}

Background: Some studies have found a negative relationship between obesity and school performance in scholars. However, this topic has not been studied in depth in Mexico. The aim of this study was to analyze the association between overweight and obesity with school performance in secondary school students from Merida. Methods: Weight and height were measured, and

Correspondencia:

*Juan Carlos Salazar-Rendón

E-mail: juan.salazar@ssy.gob.mx
Disponible en internet: 13-04-2018 Bol Med Hosp Infant Mex. 2018;75:94-103 www.bmhim.com

1665-1146/@ 2018. Hospital Infantil de México Federico Gómez, impreso por Permanyer México SA de CV, todos los derechos reservados. 
body mass index was calculated in a sample of 566 secondary students in Merida. Nutritional status was classified in normal weight $(-2-<+1$ standard deviation [SD]), overweight (+1-<2 SD) and obesity $(>+2 S D)$ according to the World Health Organization criteria. The scores in language, mathematics and sciences were used as indicators of school performance. Good performance was considered when the scores were $\geq 8$ points, and poor performance when scores were $<8$ points. Logistic regression models were used to analyse the association between the variables. Results: The $44 \%$ of the sample met the criteria for overweight and obesity. After adjusting for school grade, type of school, number of absences, and Mayan surnames, we found that girls classified as obese had a significant risk of a poor performance in mathematics (odds ratio [OR]: 3.07; 95\% confidence interval [CI]: 1.39-6.80) and language (OR: 2.55; 95\% Cl: 1.14-5.71) compared with those with healthy weight. Conclusions: Our results suggest that obesity represents a risk of having a lower school performance in girls but not in boys.

Key words: School performance. Body mass index. Obesity. Adolescents.

\section{Introducción}

La obesidad es actualmente un problema de salud pública que afecta a la población mundial de todas las edades. La obesidad en la infancia y juventud se ha relacionado con una serie de eventos adversos que aumentan los riesgos de padecer enfermedades de tipo cardiovascular, metabólicas, articulares y oncológicas durante la vida adulta ${ }^{1,2}$. Estimaciones recientes sugieren que las generaciones más jóvenes podrían tener vidas más cortas y menos saludables que la de sus padres como consecuencia de la epidemia de obesidad $^{3,4}$.

En México, la prevalencia de sobrepeso y obesidad en menores de 18 años se ha incrementado considerablemente en las últimas décadas hasta llegar a ser la más alta del mundo ${ }^{5}$. La Encuesta Nacional de Salud y Nutrición (ENSANUT) de Medio Camino 2016 señala una prevalencia combinada de sobrepeso y obesidad del $36.3 \%$ en adolescentes mexicanos de entre 12 y 19 años, predominando el sobrepeso en estudiantes de sexo femenino $(26.4 \%$ vs. $18.5 \%$ ) y la obesidad en los de sexo masculino (15\% vs. $12.8 \%)^{6}$. En el estado mexicano de Yucatán, la ENSANUT 2012 reportó una prevalencia de sobrepeso y obesidad del $43.4 \%$ en adolescentes, cifra que se ubica por encima de la media nacional, con una prevalencia ligeramente mayor en las mujeres que en los hombres (44.1\% vs. $42.7 \%)^{7}$.

La evidencia científica señala que el sobrepeso y la obesidad pueden afectar, entre otras cosas, el rendimiento académico de niños y adolescentes, lo cual sugiere que estarían asociados no solo con enfermedades crónicas, sino también con el desarrollo académico de la población en edad escolar y el capital humano en la edad adulta ${ }^{8-15}$. Al respecto, en un estudio con diseño longitudinal llevado a cabo en Tailandia, Mo-suwan, et al. reportaron una asociación negativa entre el rendimiento académico y la obesidad en la adolescencia (grados 7-9), pero no durante la niñez (grados 3-6) ${ }^{10}$. En los EE.UU., Datar y Sturm estudiaron a un grupo de alumnos desde su ingreso al nivel preescolar hasta el tercer grado de primaria, y encontraron que las niñas con obesidad y aquellas que durante este periodo pasaron de tener un peso normal a obesidad tuvieron mayor probabilidad de tener un rendimiento académico menor ${ }^{14}$. En ambos estudios, la obesidad fue determinada cuando los participantes mostraron un IMC excedido para la edad y el sexo.

Algunos autores han señalado los malos hábitos dietéticos y de sueño, el sedentarismo y los problemas emocionales como posibles mecanismos del fenómeno, así como a la discriminación hacia las personas con obesidad por parte de sus compañeros y profesores $^{11,16-18}$. Se ha propuesto que la asociación entre sobrepeso y la obesidad con el rendimiento académico vulnera la calidad de vida de los individuos, debido a que los jóvenes con obesidad tendrían mayor probabilidad de tener menos años de educación formal y menor probabilidad de conseguir empleos mejor remunerados ${ }^{19,20}$.

A pesar de haber aumentado el gasto en educación en más del $5 \%$ del producto interno bruto en los últimos años ${ }^{21}$, el sistema educativo mexicano aún enfrenta grandes retos para contribuir al desarrollo humano de su población. Los resultados obtenidos en pruebas para medir el avance educativo en el país han evidenciado niveles bajos de desempeño entre los estudiantes mexicanos ${ }^{22,23}$. La prueba Planea 2015 mostró un desempeño pobre en lenguaje y comunicación y matemáticas entre alumnos de tercero de secundaria, en los que apenas el 6.1 y el $3.1 \%$ de los participantes obtuvieron un logro satisfactorio, respectivamente ${ }^{22}$. Por su parte, la Organización para la Cooperación y el Desarrollo Económico (OCDE) aplica el Programa para la Evaluación Internacional de Alumnos de la OCDE (PISA, por sus siglas en ingles) para evaluar los logros y habilidades adquiridos por los estudiantes al final de la educación obligatoria, donde los resultados obtenidos por los 
adolescentes mexicanos ubican al país entre los más rezagados de los integrantes de la organización. Los resultados de la prueba PISA 2015 ubican a México por debajo del promedio de la OCDE en ciencias, lectura y matemáticas, donde menos del $1 \%$ de los estudiantes mexicanos lograron alcanzar niveles de excelencia; al comparar los resultados por género, los estudiantes de sexo masculino tuvieron mejores resultados en ciencias y matemáticas que los estudiantes de sexo femenino, mientras que estas superaron a los varones en lectura ${ }^{23}$.

El objetivo de este estudio fue analizar la asociación entre el sobrepeso y la obesidad con el desempeño académico en una muestra de adolescentes de 12 a 15 años que cursan la educación secundaria en la ciudad de Mérida, Yucatán.

\section{Métodos}

\section{Selección y descripción de la muestra}

Durante mayo y junio de 2016 se visitaron cinco escuelas secundarias públicas de la ciudad de Mérida, seleccionadas aleatoriamente por estrato. Tres de ellas pertenecen al subsistema de escuelas secundarias técnicas, las cuales se diferencian por incluir en su plan de estudios materias enfocadas hacia el ámbito laboral a un nivel técnico, y las restantes pertenecían al subsistema de escuelas secundarias generales. Los grupos de las escuelas fueron igualmente seleccionados de forma aleatoria. La muestra estuvo constituida por un total de 566 adolescentes (293 mujeres y 273 varones) de entre 12 y 15 años de edad.

\section{Mediciones antropométricas}

Se obtuvo el peso y la talla en los adolescentes participantes utilizando una báscula digital con precisión de $100 \mathrm{~g}$ (Omron® HBF 514c) y un estadímetro con precisión de $0.1 \mathrm{~cm}$ (Seca®213), respectivamente. Las mediciones fueron realizadas por personal capacitado siguiendo los procedimientos estandarizados sugeridos por Lohman, et al ${ }^{24}$. Los participantes fueron medidos en las escuelas estando descalzos y con ropa ligera. La talla y peso fueron utilizados para calcular el IMC (IMC = peso[kg]/talla $\left.\left[\mathrm{m}^{2}\right]\right)$ de los participantes y se calcularon los valores estandarizados (z) de este indicador utilizando los valores de referencia de la OMS de $2007^{25}$. El estado nutricional de los adolescentes fue clasificado en las siguientes categorías: peso normal $(-2-<+1 \mathrm{DE})$, sobrepeso $(+1-<2 \mathrm{DE})$ y obesidad $(\geq+2 D E)$.

\section{Medición del rendimiento académico}

La educación básica en México está conformada por los siguientes niveles: preescolar, primaria y secundaria; este último nivel tiene una duración de tres años y está dirigido a población entre los 12 a los 16 años. La escala oficial de calificaciones es numérica, del 5 al 10, siendo 6.0 la calificación mínima para aprobar. En el estado de Yucatán existen cuatro subsistemas de educación secundaria: general, estatal, técnica y telesecundaria. Para fines de este estudio, las calificaciones fueron utilizadas como un indicador del rendimiento académico de los adolescentes; se obtuvieron de los registros de las escuelas y correspondieron al cuarto bimestre (marzo-abril) del curso escolar 2015-2016. La información recabada consistió en las calificaciones de las asignaturas de lenguaje, matemáticas y ciencias. Las calificaciones fueron clasificadas en dos categorías: 1) desempeño mayor cuando fueron como mínimo de 8 puntos y 2) desempeño menor cuando fueron inferiores a 8 puntos. Si bien este punto de corte es arbitrario, la puntuación 8 representa el punto intermedio en la escala de calificaciones.

\section{Consideraciones éticas}

Se obtuvo autorización de la Secretaría de Educación del estado de Yucatán para llevar a cabo el estudio en las escuelas seleccionadas. Los investigadores informaron a los directores de las escuelas y a los adolescentes seleccionados sobre los objetivos del estudio y solicitaron su consentimiento para participar en el estudio. Se obtuvo el consentimiento informado de todos los participantes. Con el fin de mantener la privacidad de los adolescentes, las mediciones antropométricas fueron realizadas en un espacio exclusivo para esta actividad asignado por la dirección escolar. Las mediciones se llevaron a cabo en presencia únicamente del equipo de investigación.

\section{Análisis estadístico}

Las diferencias en las medias (valores absolutos y puntuaciones z) de IMC entre las categorías de desempeño académico y sexo fueron analizadas mediante pruebas de $t$ de Student no pareadas. Se aplicó la prueba de $\chi^{2}$ para analizar las diferencias en las 
proporciones de individuos en los grupos de estado nutricional de acuerdo a las categorías de desempeño académico. El análisis de la asociación entre el estado nutricional y el desempeño académico se llevó a cabo mediante modelos de regresión logística. La variable de respuesta consistió en el desempeño académico mostrado por los estudiantes $(0=$ desempeño mayor, 1 = desempeño menor). Se ajustaron modelos de regresión para predecir el desempeño en lenguaje, matemáticas y ciencias. Se desarrollaron modelos distintos para ambos sexos. La variable independiente de interés fue el estado nutricional de los estudiantes expresado por el IMC $(0=$ peso normal, $1=$ sobrepeso y 2 = obesidad). Se restringió el análisis a estas categorías dado que únicamente cinco individuos mostraron un IMC bajo (<-2 DE). Todos los modelos fueron ajustados para el grado escolar de los estudiantes ( 1 = primero, 2 = segundo y $3=$ tercero), el número de ausencias en el periodo escolar estudiado (variable continua), el tipo de escuela $(0=$ general y $1=$ técnica $)$ y la procedencia maya del apellido de los estudiantes $(0=\sin$ apellidos mayas y $1=$ uno 0 dos apellidos mayas). El tipo de escuela fue utilizado como un indicador de las condiciones socioeconómicas de los estudiantes; en el contexto local, las escuelas secundarias técnicas reciben estudiantes en condiciones socioeconómicas adversas respecto al resto de las escuelas. Estudios previos señalan que los estudiantes con ascendencia maya tienden a mostrar un desempeño académico distinto al resto de la población ${ }^{26}$.

Las categorías de IMC normal y las siguientes categorías socioeconómicas fueron usadas como referencias en la especificación de los modelos: primer grado de secundaria, escuela general y sin apellidos mayas. Todos los análisis se realizaron a un nivel de significación de $\alpha=0.05$ con el paquete estadístico Stata ${ }^{\circledR}$ (StataCorp Lp 11.2).

\section{Resultados}

La tabla 1 describe las características generales de la muestra estudiada. La media de la edad de los estudiantes fue 14.05 años (DE: 0.94) y la distribución de estos de acuerdo con su grado escolar fue 33, 43 y $24 \%$ en primer, segundo y tercer grado, respectivamente. El $56 \%$ de los participantes fue reclutado en escuelas técnicas y el $47 \%$ tuvo al menos un apellido de origen maya. Mayores proporciones (con significación estadística) de mujeres mostraron desempeño mayor en lenguaje y ciencias.
Las mujeres mostraron valores significativamente mayores de IMC que los varones (media: $22.66 \mathrm{~kg} / \mathrm{m}^{2}$ [DE: 3.91] vs. media: $21.99 \mathrm{~kg} / \mathrm{m}^{2}$ [DE: 3.92], $\mathrm{p}<0.05$ ) (Tabla 2). El 44\% de los individuos estudiados mostró sobrepeso y obesidad (sobrepeso: $28 \%$; obesidad: $16 \%$ ), siendo las cifras muy similares entre ambos sexos (hombres: 42\%; mujeres: 44\%). Las estudiantes con desempeño académico menor en matemáticas mostraron valores significativamente más altos de IMC (absoluto) que aquellas con desempeño académico mayor. Se observó también que las estudiantes con desempeño académico menor en esta área mostraron una proporción significativamente mayor de sobrepeso y obesidad en comparación a aquellas con desempeño académico alto. Este resultado no fue encontrado en varones para ningún área del conocimiento analizada.

Los resultados de los modelos de regresión logística para cada área del conocimiento se muestran en las tablas 3 a 5. Se encontró que la categoría de obesidad estuvo asociada significativamente a un mayor riesgo de tener desempeño académico menor en lenguaje (RM: 2.55; intervalo de confianza [IC] 95\%: 1.14-5.71) y matemáticas (RM: 3.07; IC 95\%: 1.39-6.80), siendo este resultado significativo en las mujeres pero no en los varones. El número de faltas de asistencia en el periodo estudiado incrementó las probabilidades de desempeño menor en todas las áreas académicas para varones y mujeres. Adicionalmente, los modelos sugirieron que los jóvenes, tanto mujeres como varones, de escuelas secundarias técnicas tuvieron mayores probabilidades de tener desempeño menor en el área de lenguaje. Ninguna interacción entre las variables independientes resultó significativa.

\section{Discusión}

La prevalencia de sobrepeso y obesidad encontrada en esta investigación (44\%) es comparable con la reportada por la ENSANUT $2012^{7}$ (43.4\%) para Yucatán en jóvenes entre 12 y 19 años y con la obtenida por la Encuesta Nacional de Salud en Escolares (ENSE) de $2008^{27}(41 \%)$ en alumnos de secundaria de Yucatán de entre 12 y 16 años de edad. Estas similitudes otorgan fiabilidad a nuestros resultados y confirman que el sobrepeso y la obesidad continúan siendo un problema de salud pública alarmante entre los adolescentes yucatecos.

Los estudios que abordan la asociación entre la obesidad y el rendimiento académico muestran resultados discrepantes; algunos estudios han encontrado 
Tabla 1. Características generales de la muestra por sexo

\begin{tabular}{|c|c|c|c|c|}
\hline \multirow[t]{2}{*}{ Variable } & Todos & Masculino & Femenino & \multirow[t]{2}{*}{ Diferencia } \\
\hline & Media (DE) & Media (DE) & Media (DE) & \\
\hline $\mathrm{n}$ & $566(100 \%)$ & $273(48 \%)$ & $293(52 \%)$ & - \\
\hline Edad & $14.05( \pm 0.94)$ & $14.13( \pm 0.93)$ & $13.98( \pm 0.95)$ & $t=1.86, p=0.064$ \\
\hline $\begin{array}{l}\text { Tipo de escuela } \\
\text { No técnica } \\
\text { Técnica }\end{array}$ & $\begin{array}{l}249(44 \%) \\
317(56 \%)\end{array}$ & $\begin{array}{l}132(48 \%) \\
141(52 \%)\end{array}$ & $\begin{array}{l}117(40 \%) \\
176(60 \%)\end{array}$ & $X^{2}{ }_{(1)}=4.06, p=0.044$ \\
\hline $\begin{array}{l}\text { Grado escolar } \\
\text { Primero } \\
\text { Segundo } \\
\text { Tercero }\end{array}$ & $\begin{array}{l}188(33 \%) \\
241(43 \%) \\
137(24 \%)\end{array}$ & $\begin{array}{c}86(32 \%) \\
118(43 \%) \\
69(25 \%)\end{array}$ & $\begin{array}{l}102(35 \%) \\
123(42 \%) \\
68(23 \%)\end{array}$ & $\mathrm{X}^{2}{ }_{(2)}=0.76, \mathrm{p}=0.681$ \\
\hline Ausencias & Mediana $=2$ & Mediana = 2 & Mediana = 2 & $z=-0.42, p=0.673$ \\
\hline $\begin{array}{l}\text { Apellidos } \\
\text { No mayas } \\
\text { Mayas }\end{array}$ & $\begin{array}{l}301(53 \%) \\
265(47 \%)\end{array}$ & $\begin{array}{l}142(52 \%) \\
131(48 \%)\end{array}$ & $\begin{array}{l}159(54 \%) \\
134(46 \%)\end{array}$ & $X^{2}{ }_{(1)}=0.29, p=0.592$ \\
\hline $\begin{array}{c}\text { Desempeño } \\
\text { Lenguaje } \\
\text { Mayor } \\
\text { Menor }\end{array}$ & $\begin{array}{l}312(55 \%) \\
253(45 \%)\end{array}$ & $\begin{array}{l}123(45 \%) \\
150(55 \%)\end{array}$ & $\begin{array}{l}189(65 \%) \\
103(35 \%)\end{array}$ & $X^{2}{ }_{(1)}=22.08, p<0.001$ \\
\hline $\begin{array}{l}\text { Matemáticas } \\
\text { Mayor } \\
\text { Menor }\end{array}$ & $\begin{array}{l}274(49 \%) \\
291(51 \%)\end{array}$ & $\begin{array}{l}122(45 \%) \\
151(55 \%)\end{array}$ & $\begin{array}{l}152(52 \%) \\
140(48 \%)\end{array}$ & $X^{2}{ }_{(1)}=3.06, p=0.080$ \\
\hline $\begin{array}{r}\text { Ciencias } \\
\text { Mayor } \\
\text { Menor }\end{array}$ & $\begin{array}{l}359(64 \%) \\
206(36 \%)\end{array}$ & $\begin{array}{l}153(56 \%) \\
120(44 \%)\end{array}$ & $\begin{array}{c}206(71 \%) \\
86(29 \%)\end{array}$ & $X^{2}{ }_{(1)}=12.81, p<0.001$ \\
\hline
\end{tabular}

DE: desviación estándar; p: grado de significación; X2: prueba de Ji cuadrado.

una asociación positiva, mientras que otros no han encontrado relación ${ }^{8-15,28-32}$. Estas discrepancias podrían deberse a las diferencias en los grupos de edad y características socioeconómicas entre las poblaciones estudiadas, así como a los diferentes métodos utilizados para evaluar el rendimiento académico en los niños y jóvenes.

En este estudio, se encontró que las mujeres con un mayor IMC mostraron mayor riesgo de tener un menor desempeño académico. Nuestros hallazgos coinciden con los reportados por Sabia ${ }^{13}$, quien encontró una relación negativa entre el IMC y las calificaciones obtenidas por mujeres blancas en los EE.UU. entre los 14-17 años de edad. Sin embargo, esta asociación perdió significación en las mujeres y varones no blancos del mismo rango de edad. Nuestros resultados también son consistentes con los reportados por Datar y Sturm, quienes realizaron un seguimiento a niños norteamericanos durante sus primeros años en educación primaria, en los que encontraron que la ganancia de peso excesiva durante los primeros años en la escuela primaria fue un factor de riesgo significativo en la obtención de resultados adversos en el desempeño académico entre las niñas, pero no en los niños ${ }^{14}$.

Los resultados muestran que las jóvenes que cumplieron los criterios de obesidad tienen mayor riesgo de tener un desempeño menor específicamente en lenguaje y matemáticas. Resultados similares han sido hallados por Judge y Jahns ${ }^{9}$, Mo-suwan, et a ${ }^{10}$ y Datar, et $\mathrm{a}^{15}$. quienes reportaron en diferentes estudios haber encontrado asociación significativa entre la obesidad y un rendimiento académico menor en matemáticas y lenguaje en niños en edad preescolar, escolar y adolescentes, independientemente del sexo. Sin embargo, en el estudio reportado por Judge y Jahns ${ }^{9}$ se encontró que la asociación se atenúa después de controlar la influencia de variables socioeconómicas y de educación materna. Otro estudio realizado con niños en educación primaria en Canadá encontró que los participantes que habían presentado obesidad en edad preescolar y habían conseguido un peso saludable en la primaria lograron mejorar sus calificaciones en matemáticas ${ }^{32}$. 
Tabla 2. Estadística descriptiva del IMC y la proporción de individuos en categorías de estado nutricional según categorías de desempeño académico en lenguaje, matemáticas y ciencias

\begin{tabular}{|c|c|c|c|c|c|c|c|}
\hline \multirow[t]{3}{*}{ Indicador } & \multirow[t]{2}{*}{ Todos } & \multicolumn{2}{|c|}{ Lenguaje } & \multicolumn{2}{|c|}{ Matemáticas } & \multicolumn{2}{|c|}{ Ciencias } \\
\hline & & Mayor & Menor & Mayor & Menor & Mayor & Menor \\
\hline & Media (DE) & Media (DE) & Media (DE) & Media (DE) & Media (DE) & Media (DE) & Media (DE) \\
\hline \multicolumn{8}{|l|}{ Mujeres } \\
\hline \multirow[t]{2}{*}{ IMC $\left(\mathrm{kg} / \mathrm{m}^{2}\right)$} & $22.66(3.91)$ & $22.38(3.86)$ & $23.23(3.96)$ & $22.17(3.46)$ & $23.24(4.27)$ & $22.45(3.87)$ & 23.24 (3.95) \\
\hline & & \multicolumn{2}{|c|}{$t=-1.78, p=0.060$} & \multicolumn{2}{|c|}{$\mathrm{t}=-2.36, \mathrm{p}=0.019$} & \multicolumn{2}{|c|}{$t=-1.58, p=0.115$} \\
\hline \multirow[t]{2}{*}{ IMC-Z } & $0.58(0.79)$ & $0.77(1.03)$ & $0.95(0.99)$ & $0.72(0.93)$ & $0.95(1.10)$ & $0.78(1.02)$ & $0.96(1.01)$ \\
\hline & & \multicolumn{2}{|c|}{$\mathrm{t}=-1.47, \mathrm{p}=0.141$} & \multicolumn{2}{|c|}{$\mathrm{t}=-1.87, \mathrm{p}=0.061$} & \multicolumn{2}{|c|}{$t=-1.78, p=0.060$} \\
\hline \multirow[t]{2}{*}{$\begin{array}{l}\text { IMC } \\
\text { Normal } \\
\text { Sobrepeso } \\
\text { Obesidad }\end{array}$} & \multirow[t]{2}{*}{$\begin{array}{c}56 \%(164) \\
30 \%(87) \\
14 \%(41)\end{array}$} & $\begin{array}{l}58 \%(109) \\
31 \%(58) \\
11 \%(21)\end{array}$ & $\begin{array}{l}53 \%(54) \\
28 \%(29) \\
19 \%(20)\end{array}$ & $\begin{array}{c}59 \%(90) \\
32 \%(48) \\
9 \%(13)\end{array}$ & $\begin{array}{l}52 \%(73) \\
28 \%(39) \\
20 \%(28)\end{array}$ & $\begin{array}{c}58 \%(119) \\
28 \%(58) \\
14 \%(28)\end{array}$ & $\begin{array}{l}51 \%(44) \\
34 \%(29) \\
15 \%(13)\end{array}$ \\
\hline & & \multicolumn{2}{|c|}{$X^{2}=3.74, p=0.154$} & \multicolumn{2}{|c|}{$X^{2}=7.79, p=0.020$} & \multicolumn{2}{|c|}{$X^{2}=1.20, p=0.548$} \\
\hline \multicolumn{8}{|l|}{ Hombres } \\
\hline \multirow[t]{2}{*}{ IMC $\left(\mathrm{kg} / \mathrm{m}^{2}\right)$} & $21.99(3.92)$ & $21.76(3.81)$ & $22.17(4.02)$ & $21.80(3.80)$ & $22.13(4.02)$ & $21.83(3.99)$ & $22.18(3.84)$ \\
\hline & & \multicolumn{2}{|c|}{$t=-0.86, p=0.391$} & \multicolumn{2}{|c|}{$\mathrm{t}=-0.69, \mathrm{p}=0.491$} & \multicolumn{2}{|c|}{$t=-0.73, p=0.466$} \\
\hline \multirow[t]{2}{*}{ IMC-Z } & $0.56(0.99)$ & $0.73(1.18)$ & $0.83(1.19)$ & $0.71(1.18)$ & $0.84(1.20)$ & $0.73(1.21)$ & $0.85(1.17)$ \\
\hline & & \multicolumn{2}{|c|}{$\mathrm{t}=-0.64, \mathrm{p}=0.520$} & \multicolumn{2}{|c|}{$t=-0.89, p=0.373$} & \multicolumn{2}{|c|}{$t=-0.87, p=0.385$} \\
\hline \multirow[t]{2}{*}{$\begin{array}{l}\text { IMC } \\
\text { Normal } \\
\text { Sobrepeso } \\
\text { Obesidad }\end{array}$} & \multirow[t]{2}{*}{$\begin{array}{c}57 \%(153) \\
26 \%(69) \\
16 \%(47)\end{array}$} & $\begin{array}{l}57 \%(68) \\
27 \%(33) \\
16 \%(19)\end{array}$ & $\begin{array}{l}57 \%(85) \\
24 \%(36) \\
19 \%(28)\end{array}$ & $\begin{array}{l}56 \%(66) \\
30 \%(36) \\
14 \%(17)\end{array}$ & $\begin{array}{l}58 \%(87) \\
22 \%(33) \\
20 \%(30)\end{array}$ & $\begin{array}{l}57 \%(86) \\
25 \%(37) \\
18 \%(27)\end{array}$ & $\begin{array}{l}56 \%(67) \\
27 \%(32) \\
17 \%(20)\end{array}$ \\
\hline & & \multicolumn{2}{|c|}{$X^{2}=0.62, p=0.732$} & \multicolumn{2}{|c|}{$X^{2}=3.08, p=0.215$} & \multicolumn{2}{|c|}{$X^{2}=0.19, p=0.907$} \\
\hline
\end{tabular}

DE: desviación estándar; p: grado de significación; t: prueba t de Student; IMC: índice de masa corporal; IMC-Z: puntuación z del índice de masa corporal, normal $(-2-<+1 D E)$, sobrepeso $(+1-<2 D E)$, obesidad $(\geq+2 D E)$.

A diferencia de nuestros hallazgos, estos estudios no reportan si sus resultados variaron de acuerdo al sexo de los participantes.

Los posibles mecanismos que explican la relación entre el sobrepeso y la obesidad con el rendimiento académico aún no son claros. La literatura muestra una asociación entre la función cognitiva y la adiposidad, en la que funciones de control cognitivo, capacidades de función visoespacial y habilidad mental se ven alteradas en los niños con adiposidad excesiva ${ }^{33,34}$. En este sentido, Portillo-Reyes et al. analizaron el rendimiento neuropsicológico y estado nutricional en una muestra de niños mexicanos entre 8 y 12 años de bajo nivel socioeconómico y pertenecientes a entornos urbanos. Los resultados mostraron que los niños con obesidad presentaban un menor rendimiento que los niños con peso saludable en las áreas de memoria, razonamiento abstracto y seguimiento de instrucciones ${ }^{35}$.

Los hábitos de salud como la dieta, la actividad física y el sueño, así como factores psicosociales, han sido propuestos como vínculos entre el sobrepeso y obesidad con un menor rendimiento académico en la infancia y la juventud ${ }^{11}$. Estudios realizados en Canadá y Corea han encontrado que el hábito de desayunar y consumir una dieta saludable son factores asociados a un mayor rendimiento académico en niños y adolescentes ${ }^{36-38}$. Particularmente, Kang y Park reportaron que los adolescentes que no desayunaban tenían menores calificaciones en lenguaje, matemáticas y lengua extranjera en comparación con sus compañeros que sí lo hacían ${ }^{38}$. Haapala et al. señalan que el 
Tabla 3. Modelo de regresión logística de factores biosociales para desempeño menor en lenguaje

\begin{tabular}{|c|c|c|c|c|c|c|c|c|}
\hline & \multicolumn{4}{|c|}{ Mujeres } & \multicolumn{4}{|c|}{ Hombres } \\
\hline & $n$ & $\begin{array}{l}\text { RM (IC 95\%) } \\
\text { crudas }\end{array}$ & $\begin{array}{c}\text { RM (IC 95\%) } \\
\text { ajustadas }\end{array}$ & Valor $\mathbf{p}$ & $n$ & $\begin{array}{l}\text { RM (IC 95\%) } \\
\text { crudas }\end{array}$ & $\begin{array}{c}\text { RM (IC 95\%) } \\
\text { ajustadas }\end{array}$ & Valor $p$ \\
\hline $\begin{array}{l}\text { IMC } \\
\text { Normal } \\
\text { Sobrepeso } \\
\text { Obesidad }\end{array}$ & $\begin{array}{c}164 \\
87 \\
41\end{array}$ & $\begin{array}{c}1 \\
1.01(0.58-1.75) \\
1.92(0.96-3.85)\end{array}$ & $\begin{array}{c}1 \\
1.19(0.63-2.26) \\
2.55(1.14-5.71)\end{array}$ & $\begin{array}{c}- \\
0.588 \\
0.023\end{array}$ & $\begin{array}{c}153 \\
69 \\
47\end{array}$ & $\begin{array}{c}1 \\
0.87(0.49-1.54) \\
1.18(0.61-2.29)\end{array}$ & $\begin{array}{c}1 \\
0.83(0.44-1.54) \\
1.13(0.54-2.36)\end{array}$ & $\begin{array}{c}- \\
0.547 \\
0.738\end{array}$ \\
\hline $\begin{array}{l}\text { Grado escolar } \\
\text { 1. }{ }^{\text {er }} \text { grado } \\
2 .^{\text {do }} \text { grado } \\
\text { 3. }{ }^{\text {er }} \text { grado } \\
\text { Ausencias }\end{array}$ & $\begin{array}{c}101 \\
123 \\
68 \\
292\end{array}$ & $\begin{array}{c}1 \\
2.10(1.18-3.72) \\
1.81(0.93-3.51) \\
1.24(1.16-1.33)\end{array}$ & $\begin{array}{c}1 \\
2.13(1.10-4.14) \\
1.11(0.52-2.37) \\
1.26(1.17-1.36)\end{array}$ & $\begin{array}{c}- \\
0.025 \\
0.794 \\
<0.001\end{array}$ & $\begin{array}{c}85 \\
115 \\
69 \\
269\end{array}$ & $\begin{array}{c}1 \\
0.85(0.48-1.48) \\
1.16(0.61-2.20) \\
1.19(1.10-1.29)\end{array}$ & $\begin{array}{c}1 \\
1.07(0.58-2.00) \\
0.76(0.37-1.58) \\
1.21(1.12-1.32)\end{array}$ & $\begin{array}{c}- \\
0.823 \\
0.465 \\
<0.001\end{array}$ \\
\hline $\begin{array}{l}\text { Tipo de escuela } \\
\text { No técnica } \\
\text { Técnica }\end{array}$ & $\begin{array}{l}116 \\
176\end{array}$ & $\begin{array}{c}1 \\
2.75(1.62-4.66)\end{array}$ & $\begin{array}{c}1 \\
3.03(1.64-5.62)\end{array}$ & $<0.001$ & $\begin{array}{l}129 \\
140\end{array}$ & $\begin{array}{c}1 \\
2.12(1.30-3.44)\end{array}$ & $\begin{array}{c}1 \\
2.19(1.28-3.76)\end{array}$ & 0.004 \\
\hline $\begin{array}{l}\text { Apellidos } \\
\text { No maya } \\
\text { Maya }\end{array}$ & $\begin{array}{l}158 \\
134\end{array}$ & $\begin{array}{c}1 \\
1.05(0.65-1.69)\end{array}$ & $\begin{array}{c}1 \\
0.93(0.52-1.66)\end{array}$ & $0 . \overline{-}$ & $\begin{array}{l}138 \\
131\end{array}$ & $\frac{1}{1.52(0.94-2.46)}$ & $\begin{array}{c}1 \\
1.58(0.93-2.67)\end{array}$ & 0.089 \\
\hline
\end{tabular}

DE: desviación estándar; p: grado de significación; IC: intervalo de confianza.

Normal $(-2-<+1 \mathrm{DE})$; Sobrepeso $(+1-<2 \mathrm{DE})$, Obesidad $(\geq+2 \mathrm{DE})$.

El modelo fue ajustado para las siguientes variables: grado escolar, número de ausencias durante el bimestre estudiado, tipo de escuela y ascendencia maya del estudiante de acuerdo a la presencia de apellidos mayas.

consumo de determinadas dietas, como la mediterránea o la báltica, se asoció con mejores habilidades de lectura, pero no con mejores habilidades aritméticas entre niños finlandeses que cursaban los primeros años de la escuela primaria ${ }^{39}$.

Respecto a los hábitos de sueño, un estudio realizado en México analizó el desempeño académico de un grupo de mujeres adolescentes entre los 15 y 18 años de edad, encontrando que aquellas participantes que cubrieron los criterios de obesidad y que además presentaron hipersomnia diurna tuvieron un rendimiento académico un $0.7 \%$ menor que sus pares con peso saludable y sin trastornos del sueño ${ }^{16}$.

Los beneficios de la actividad física parecen extenderse hasta el rendimiento académico, como sugieren Kantomaa et al., quienes encontraron que los adolescentes físicamente activos tenían el doble de probabilidades de tener mejores calificaciones comparados con los adolescentes sedentarios ${ }^{40}$. Por su parte, CarIson, et al. ${ }^{41}$ reportaron que un mayor tiempo de practica de actividad física (70-300 min/semana) se asociaba con un beneficio académico en matemáticas y lectura en niñas norteamericanas que cursaban la primaria. Otro estudio realizado en EE.UU, con alumnos que, específicamente, cursaban el tercer y quinto grado en escuelas públicas, reportó que la capacidad aeróbica estaba positivamente asociada con el rendimiento académico en general y en las áreas de matemáticas y lectura ${ }^{42}$. Estos beneficios podrían estar regulados por la producción de endorfinas, las cuales mejoran el estado de ánimo y a la socialización que conlleva la práctica de deportes.

Cierta evidencia respalda el papel de los factores psicosociales en el rendimiento académico. Se ha reportado que los niños que presentan obesidad sufren frecuentemente de burla y mayor acoso escolar (bullying) que sus compañeros; este acoso podría disminuir su autoestima, y en consecuencia afectar potencialmente su rendimiento académico ${ }^{43-45}$. Es posible que la influencia del sobrepeso y la obesidad sobre el rendimiento académico se manifieste en mayor medida en las mujeres. Esta relación parece tener consecuencias que afectan tanto su educación como el empleo, el cuidado de su salud y las relaciones interpersonales ${ }^{46,47}$. Crosnoe encontró que las niñas obesas tenían menos posibilidades de asistir a la universidad después de terminar la escuela preparatoria, a diferencia de sus compañeras con un peso saludable; esta diferencia no se observó entre los hombres con sobrepeso y obesidad ${ }^{48}$.

Este estudio tiene algunas limitaciones que deben ser discutidas. Al ser un estudio de corte transversal, no pretende señalar que la obesidad tenga un efecto de causalidad en el desempeño académico, sino que 
Tabla 4. Modelo de regresión logística de factores biosociales para desempeño menor en matemáticas

\begin{tabular}{|c|c|c|c|c|c|c|c|c|}
\hline & \multicolumn{4}{|c|}{ Mujeres } & \multicolumn{4}{|c|}{ Hombres } \\
\hline & $\mathbf{n}$ & $\begin{array}{c}\text { RM (IC 95\%) } \\
\text { crudas }\end{array}$ & $\begin{array}{c}\text { RM (IC 95\%) } \\
\text { ajustadas }\end{array}$ & Valor $p$ & $\mathbf{n}$ & $\begin{array}{c}\text { RM (IC 95\%) } \\
\text { crudas }\end{array}$ & $\begin{array}{l}\text { RM (IC 95\%) } \\
\text { ajustadas }\end{array}$ & Valor $p$ \\
\hline $\begin{array}{l}\text { IMC } \\
\text { Normal } \\
\text { Sobrepeso } \\
\text { Obesidad }\end{array}$ & $\begin{array}{c}164 \\
87 \\
41\end{array}$ & $\begin{array}{c}1 \\
1.01(0.59-1.69) \\
2.66(1.28-5.49)\end{array}$ & $\begin{array}{c}1 \\
1.07(0.61-1.89) \\
3.07(1.39-6.80)\end{array}$ & $\begin{array}{c}- \\
0.876 \\
0.006\end{array}$ & $\begin{array}{c}153 \\
69 \\
47\end{array}$ & $\begin{array}{c}1 \\
0.70(0.40-1.23) \\
1.34(0.68-2.63)\end{array}$ & $\begin{array}{c}1 \\
0.59(0.32-1.11) \\
1.05(0.51-2.18)\end{array}$ & $\begin{array}{c}- \\
0.101 \\
0.899\end{array}$ \\
\hline $\begin{array}{l}\text { Grado escolar } \\
1^{\text {er }} \text { grado } \\
2^{\text {do }} \text { grado } \\
3^{\text {er }} \text { grado } \\
\text { Ausencias }\end{array}$ & $\begin{array}{c}101 \\
123 \\
68 \\
292\end{array}$ & $\begin{array}{c}1 \\
1.21(0.71-2.05) \\
0.98(0.53-1.82) \\
1.21(1.13-1.29)\end{array}$ & $\begin{array}{c}1 \\
1.08(0.60-1.92) \\
0.56(0.28-1.13) \\
1.24(1.15-1.34)\end{array}$ & $\begin{array}{c}- \\
0.802 \\
0.104 \\
<0.001\end{array}$ & $\begin{array}{c}85 \\
115 \\
69 \\
269\end{array}$ & $\begin{array}{c}1 \\
0.78(0.44-1.38) \\
0.46(0.24-0.87) \\
1.15(1.07-1.23)\end{array}$ & $\begin{array}{c}1 \\
0.86(0.47-1.60) \\
0.28(0.12-0.55) \\
1.20(1.11-1.30)\end{array}$ & $\begin{array}{c}- \\
0.643 \\
<0.001 \\
<0.001\end{array}$ \\
\hline $\begin{array}{l}\text { Tipo de escuela } \\
\text { No técnica } \\
\text { Técnica }\end{array}$ & $\begin{array}{l}116 \\
176\end{array}$ & $\begin{array}{c}1 \\
1.01(0.63-1.61)\end{array}$ & $\begin{array}{c}1 \\
0.86(0.51-1.45)\end{array}$ & $\begin{array}{c}- \\
0.596\end{array}$ & $\begin{array}{l}129 \\
140\end{array}$ & $\begin{array}{c}1 \\
1.27(0.79-2.05)\end{array}$ & $\begin{array}{c}1 \\
1.38(0.81-2.36)\end{array}$ & $0 . \overline{243}$ \\
\hline $\begin{array}{l}\text { Apellidos } \\
\text { No maya } \\
\text { Maya }\end{array}$ & $\begin{array}{l}158 \\
134\end{array}$ & $\begin{array}{c}1 \\
1.04(0.66-1.65)\end{array}$ & $\begin{array}{c}1 \\
1.10(0.65-1.84)\end{array}$ & 0.732 & $\begin{array}{l}138 \\
131\end{array}$ & $\begin{array}{c}1 \\
1.31(0.81-2.12)\end{array}$ & $\begin{array}{c}1 \\
1.44(0.85-2.44)\end{array}$ & $0 . \overline{175}$ \\
\hline
\end{tabular}

RM: razón de momios; p: grado de significación; IC: intervalo de confianza.

Normal $(-2-<+1 \mathrm{DE})$; Sobrepeso $(+1-<2 \mathrm{DE})$, Obesidad $(\geq+2 \mathrm{DE})$.

El modelo fue ajustado para las siguientes variables: grado escolar, número de ausencias durante el bimestre estudiado, tipo de escuela y ascendencia maya del estudiante de acuerdo a la presencia de apellidos mayas.

Tabla 5. Modelo de regresión logística de factores biosociales para desempeño menor en ciencias

\begin{tabular}{|c|c|c|c|c|c|c|c|c|}
\hline & \multicolumn{4}{|c|}{ Mujeres } & \multicolumn{4}{|c|}{ Hombres } \\
\hline & $\mathbf{n}$ & $\begin{array}{c}\text { RM (IC 95\%) } \\
\text { crudas }\end{array}$ & $\begin{array}{l}\text { RM (IC 95\%) } \\
\text { ajustadas }\end{array}$ & Valor $\mathbf{p}$ & $n$ & $\begin{array}{l}\text { RM (IC 95\%) } \\
\text { crudas }\end{array}$ & $\begin{array}{l}\text { RM (IC 95\%) } \\
\text { ajustadas }\end{array}$ & Valor $\mathbf{p}$ \\
\hline $\begin{array}{l}\text { IMC } \\
\text { Normal } \\
\text { Sobrepeso } \\
\text { Obesidad }\end{array}$ & $\begin{array}{l}164 \\
87 \\
41\end{array}$ & $\begin{array}{c}1 \\
1.35(0.77-2.38) \\
1.26(0.60-2.64)\end{array}$ & $\begin{array}{c}1 \\
1.46(0.80-2.67) \\
1.22(0.54-2.73)\end{array}$ & $\begin{array}{c}- \\
0.222 \\
0.630\end{array}$ & $\begin{array}{l}153 \\
69 \\
47\end{array}$ & $\begin{array}{c}1 \\
1.11(0.63-1.96) \\
0.95(0.49-1.84)\end{array}$ & $\begin{array}{c}1 \\
1.25(0.66-2.37) \\
1.07(0.51-2.23)\end{array}$ & $\begin{array}{c}- \\
0.488 \\
0.859\end{array}$ \\
\hline $\begin{array}{l}\text { Grado escolar } \\
\text { 1. }{ }^{\text {er }} \text { grado } \\
\text { 2. }^{\text {do }} \text { grado } \\
\text { 3. er grado } \\
\text { Ausencias }\end{array}$ & $\begin{array}{c}101 \\
123 \\
68 \\
292\end{array}$ & $\begin{array}{c}1 \\
1.36(0.75-2.48) \\
2.01(1.03-3.93) \\
1.14(1.08-1.20)\end{array}$ & $\begin{array}{c}1 \\
1.13(0.59-2.14) \\
1.59(0.78-3.23) \\
1.14(1.08-1.20)\end{array}$ & $\begin{array}{c}- \\
0.718 \\
0.202 \\
<0.001\end{array}$ & $\begin{array}{c}85 \\
115 \\
69 \\
269\end{array}$ & $\begin{array}{c}1 \\
1.86(1.06-3.29) \\
1.02(0.53-1.96) \\
1.18(1.10-1.26)\end{array}$ & $\begin{array}{c}1 \\
2.01(1.07-3.79) \\
0.64(0.29-1.39) \\
1.24(1.15-1.34)\end{array}$ & $\begin{array}{c}- \\
0.030 \\
0.256 \\
<0.001\end{array}$ \\
\hline $\begin{array}{l}\text { Tipo de escuela } \\
\text { No técnica } \\
\text { Técnica }\end{array}$ & $\begin{array}{l}116 \\
176\end{array}$ & $\begin{array}{c}1 \\
0.84(0.50-1.40)\end{array}$ & $\begin{array}{c}1 \\
0.77(0.44-1.35)\end{array}$ & 0.364 & $\begin{array}{l}129 \\
140\end{array}$ & $\begin{array}{c}1 \\
0.46(0.28-0.74)\end{array}$ & $\begin{array}{c}1 \\
0.44(0.25-0.77)\end{array}$ & 0.004 \\
\hline $\begin{array}{l}\text { Apellidos } \\
\text { No maya } \\
\text { Maya }\end{array}$ & $\begin{array}{l}158 \\
134\end{array}$ & $\begin{array}{c}1 \\
0.56(0.34-0.95)\end{array}$ & $\begin{array}{c}1 \\
0.59(0.34-1.03)\end{array}$ & $\begin{array}{c}- \\
0.064\end{array}$ & $\begin{array}{l}138 \\
131\end{array}$ & $\begin{array}{c}1 \\
0.86(0.53-1.38)\end{array}$ & $\begin{array}{c}1 \\
0.94(0.55-1.61)\end{array}$ & $\begin{array}{c}- \\
0.813\end{array}$ \\
\hline
\end{tabular}

RM: razón de momios; p: grado de significación; IC: intervalo de confianza.

Normal $(-2-<+1 \mathrm{DE})$; Sobrepeso $(+1-<2 \mathrm{DE})$, Obesidad $(\geq+2 \mathrm{DE})$.

El modelo fue ajustado para las siguientes variables: grado escolar, número de ausencias durante el bimestre estudiado, tipo de escuela y ascendencia maya del estudiante de acuerdo a la presencia de apellidos mayas.

ambos valores se hallaron asociados. Por otro lado, las calificaciones escolares analizadas pertenecen únicamente a un bimestre del ciclo escolar, por lo que no representan el desempeño global de los estudiantes.
Asimismo, las calificaciones fueron asignadas por el personal docente de las escuelas, por lo cual no puede rechazarse la posibilidad de algún sesgo inherente a la evaluación y criterio de los profesores. 
En síntesis, los presentes resultados mostraron que las niñas con obesidad tuvieron riesgos incrementados de tener desempeño menor en las áreas de lenguaje y matemáticas. Estos resultados no se observaron en varones en ningún área analizada. Es necesario analizar en el futuro el papel de otros factores, como los socioeconómicos, los dietéticos y la actividad física, en el rendimiento académico de la población escolar con la finalidad de implementar políticas orientadas a mejorar sus condiciones de salud e incrementar las posibilidades de desarrollo humano.

\section{Responsabilidades éticas}

Protección de personas y animales. Los autores declaran que para esta investigación no se han realizado experimentos en seres humanos ni en animales.

Confidencialidad de los datos. Los autores declaran que han seguido los protocolos de su centro de trabajo sobre la publicación de datos de pacientes.

Derecho a la privacidad y consentimiento informado. Los autores han obtenido el consentimiento informado de los pacientes y/o sujetos referidos en el artículo. Este documento obra en poder del autor de correspondencia.

\section{Conflicto de intereses}

Los autores declaran no tener ningún conflicto de intereses

\section{Agradecimientos}

Los autores manifestamos nuestra gratitud al personal administrativo de las escuelas que participaron en este estudio por todas las facilidades otorgadas para poder realizar nuestro trabajo. Agradecemos también a todos los alumnos participantes su apoyo voluntario. De igual manera, expresamos nuestro agradecimiento a la Licenciada en Nutrición María José Peraza Ávila del Departamento de Desarrollo Humano de la SEGEY y a Ligia Martín, María Fernanda Rivero y Arantxa Ibarra, estudiantes de la Licenciatura en Nutrición de la Universidad Modelo, por su valiosa colaboración durante el trabajo de campo.

\section{Referencias}

1. Daniels SR. The consequences of childhood overweight and obesity. Future Child. 2006;16:47-67.

2. Biro FM, Wien M. Childhood obesity and adult morbidities. Am J Clin Nutr. 2010;91:1499S-505S.
3. Fontaine KR, Redden DT, Wang C, Westfall AO, Allison DB. Years of life lost due to obesity. JAMA. 2003;289:187-93.

4. Olshansky SJ, Passaro DJ, Hershow RC, Layden J, Carnes BA, Brody J, et al. A potential decline in life expectancy in the United States in the $21^{\text {st }}$ century. N Eng J Med. 2005;352:1138-45.

5. Bonvecchio A, Safdie M, Monterrubio EA, Gust T, Villalpando S, Rivera JA. Overweight and obesity trends in Mexican children 2 to 18 years of age from 1988 to 2006. Salud Publica Mex. 2009;51:S586-94.

6. Instituto Nacional de Salud Pública. Encuesta Nacional de Salud y Nutrición de Medio Camino 2016. Cuernavaca, México: Instituto Nacional de Salud Pública; 2016.

7. Instituto Nacional de Salud Pública. Encuesta Nacional de Salud y Nutrición 2012. Resultados por entidad federativa, Yucatán [Internet]. Cuernavaca, México: Instituto Nacional de Salud Pública; 2013. Disponible en: encuestas.insp.mx

8. Taras H, Potts-Datema. Obesity and student performance at school. J Sch Healt. 2005;75:291-5.

9. Judge $S$, Jahns L. Association of overweight with academic performance and social and behavioral problems: and update from the early childhood longitudinal study. J Sch Healt. 2007;77:672-8.

10. Mo-suwan L, Lebel L, Puetpaiboon A, Junjana C. School performance and weight status of children and young adolescents in a transitional society in Thailand. Int J of Obes. 1999;23:272-7.

11. Sigfúsdóttir ID, Kristjánsson AL, Allegrante JP. Health behavior and academic achievement in Icelandic school children. Health Educ Res. 2007;22:70-80.

12. Shore SM, Sachs ML, Lidicker JR, Brett SN, Wright AR, Libonati JR. Decreased scholastic achievement in overweight middle school students. Obesity. 2008;16:1535-8.

13. Sabia JJ. The effect of body weight on adolescent academic performance. South Econ J. 2007;73:871-900.

14. Datar A, Sturm R. Childhood overweight and elementary school outcomes. Int J Obes. 2006;30:1449-60.

15. Datar A, Sturm R, Magnabosco JL. Childhood overweight and academic performance: national study of kindergartens and first-graders. Obes Res. 2004;12:58-68

16. Lombardo-Aburto E, Velázquez-Moctezuma J, Flores-Rojas G, Casillas-Vaillard GA, Galvan-López A, García-Valdéz P, et al. Relación entre trastornos del sueño, rendimiento académico y obesidad en estudiantes de preparatoria. Acta Pediatr Mex. 2011;32:163-8.

17. Sikorski C, Luppa M, Brähler E, König HH, Riedel-Heller SG. Obese children, adults and senior citizens in the eyes of the general public: results of a representative on stigma and causation of obesity. PLoS One. 2012;7:e46924.

18. MacCann C, Roberts RD. Just smart but not as successful: obese students obtain lower school grades but equivalent test scores to nonobese students. Int J Obes. 2013;37:40-6.

19. Gortmarker SL, Must A, Perrin JM, Sobol AM, Dietz WH. Social and economic consequences of overweight in adolescence and young adulthood. N Engl J Med. 1993;329:1008-12.

20. Cawley J. The impact of obesity on wages. J Hum Resour. 2004;39:451-74.

21. Organización para la Cooperación y el Desarrollo Económico [Internet]. México: Panorama de la educación [Fecha consulta: 6 ene 2017]. Disponible en: http://www.oecd.org/mexico/Mexico-EAG2014-Country-Note-spanish.pdfhttp://www.oecd.org/mexico/Mexico-EAG2014-Country-Note-spanish.pdf.

22. Instituto Nacional para la Evaluación de la Educación (INEE) [Internet] Plan Nacional para la Evaluación de los aprendizajes (Planea). Resultados nacionales 2015 [Fecha consulta: 6 ene 2017]. Disponible en: http:// www.inee.edu.mx/images/stories/2015/planea/final/fasciculos-finales/resultadosPlanea-3011.pdf.

23. Organización para la Cooperación y el Desarrollo Económico [Internet]. Programa para la evaluación internacional de alumnos (PISA) PISA 2015 - resultados México [Fecha de consulta: 6 ene 2017]. Disponible en: https://www.oecd.org/pisa/PISA-2015-Mexico-ESP.pdf.

24. Lohman T, Roche A, Martorell R. Anthropometric standardization reference manual. Champaing: Human Kinetics Books; 1991.

25. Onis MD, Onyango AW, Borghi E, Siyam A, Nishida C, Siekmann J. Development of WHO growth reference for school-aged children and adolescents. Bull World Health Organ. 2007;85:660-7.

26. Mendez N, Vera-Avilez M, Gonzalez-Herrera L, Dickinson F, Palma-Solis M, Prelip M. School grades and standardized test scores of children with and without obesity in south-east Mexico. Int J School Health. 2017;4:e36356.

27. Shama-Levy T, editora. Encuesta Nacional de Salud en Escolares 2008. Cuernavaca: Instituto Nacional de Salud Pública (MX); 2010.

28. Kaestner R, Grossman M. Effects of weight on children's educational achievement. Econ Educ Rev. 2009;28:651-61.

29. Barrigas C, Fragoso I. Obesity, academic performance and reasoning ability in Portuguese students between 6 and 12 years old. J Biosoc Sci. 2012;44:165-79.

30. Flores-Saldaña B, Guízar-Mendoza JM. Obesidad infantil y aprovechamiento escolar: ¿existe relación entre obesidad y aprovechamiento escolar? Rev Hosp Jua Mex. 2009;76:137-43. 
31. Abdelalim A, Aiaj N, Al-Tmimy A, Alyousefi M, Al-Rashaidan S, Hammoud M, et al. Childhood obesity and academic achievement among male students in public primary schools in Kuwait. Med Princ Pract. 2012;21:14-9.

32. Carter MA, Dubois L, Ramsay T. Examining the relationship between obesity and math performance among Canadian school children: a prospective analysis. Int J Pediatr Obes. 2010;5:412-9.

33. Li Y, Dai Q, Jackson JC, Zhang J. Overweight is associated with decreased cognitive functioning among school-age children and adolescents. Obesity. 2008;16:1809-15.

34. Kamijo K, Khan NA, Pontifex MB, Scudder MR, Drollette ES, Raine LB, et al. The relationship of adiposity to cognitive control and scholastic achievement in preadolescent children. Obesity. 2012;20:2406-11.

35. Portillo-Reyes V, Puente AE, Pérez-García M, Loya-Méndez Y. Deterioro neuropsicológico en niños mexicanos con estados nutricionales alterados: desnutrición leve a moderada vs. obesidad. Revista Neuropsicología, Neuropsiquiatría y Neurociencias. 2011;11:133-46.

36. Florence MD, Asbridge M, Veugelers PJ. Diet quality and academic performance. J Sch Health. 2008;78:209-15.

37. Kim HYP, Frongillo EA, Han SS, Oh SY, Kim WK, Jang YA, et al. Academic performance of Korean children is associated with dietary behaviours and physical status. Asia Pacific J Clin Nutr. 2003;12:186-92.

38. Kang YW, Park JH. Does skipping breakfast and being overweight influence academic achievement among Korean adolescents? Osong Public Health Res Perspect. 2016;7:220-7.
39. Haapala EA, Eloranta AM, Venäläinen $T$, Jalkanen $H$, Poikkeus AM, Ahonen $\mathrm{T}$, et al. Diet quality and academic achievement: a prospective study among primary school children. Eur J Nutr. 2017;56:2299-308.

40. Kantomaa MT, Stamatakis E, Kankaanpää A, Kajantie E, Taanila A, Tammelin T. Association of physical activity and sedentary behavior with adolescent academic achievement. J Res Adolesc. 2015;26:432-42.

41. Carlson SA, Fulton JE, Lee SM, Maynard M, Brown DR, Kohl HW, et al. Physical education and academic achievement in elementary school: data from the Early Childhood Longitudinal Study. Am J Public Health. 2008:98:721-7.

42. Castelli DM, Hillman $\mathrm{CH}$, Buck SM, Erwin HE. Physical fitness and academic achievement in third- and fifth-grade students. J Sport Excer Psychol. 2007;29:239-52.

43. Krukowski RA, Smith West D, Philyaw Perez A, Bursac Z, Philips MM Raczynski JM. Overweight children, weight-based teasing and academic performance. Int J Pediatr Obes. 2009;4:274-80.

44. Puhl RM, King KM. Weight discrimination and bullying. Best Pract Res Clin Endocrinol Metab. 2013:27:117-27.

45. Martin-Nemeth PA, Penckofer S. Psychological vulnerability among overweight/obese minority adolescents. J Sch Nurs. 2012;28:291-301.

46. Fikkan JL, Rothblum ED. Is fat a feminist issue? Exploring the gendered nature of weight bias. Sex Roles. 2012;66:575-92.

47. Chrisler JC. "Why can't you control yourself?" fat should be a feminist issue. Sex Roles. 2012;66:608-16.

48. Crosnoe R. Gender, obesity and education. Sociol Educ. 2007;80:241-60. 